\title{
Vaincre l'enfer sur scène. Les diableries dans le Mystère des Actes des apôtres
}

Mémoire de master en histoire et littérature, sous la direction d'Isabel Iribarren et de Thierry Revol. Université de Strasbourg. Soutenu à l'université de Strasbourg le 3 septembre 2015

\section{Mathilde Cornu}

\section{(2) OpenEdition}

1 Journals

\section{Édition électronique}

URL : https://journals.openedition.org/cem/14575

DOI : $10.4000 /$ cem. 14575

ISSN : 1954-3093

Éditeur

Centre d'études médiévales Saint-Germain d'Auxerre

\section{Référence électronique}

Mathilde Cornu, "Vaincre l'enfer sur scène. Les diableries dans le Mystère des Actes des apôtres », Bulletin du centre d'études médiévales d'Auxerre | BUCEMA [En ligne], 20.2 | 2016, mis en ligne le 06 mars 2017, consulté le 22 septembre 2022. URL : http://journals.openedition.org/cem/14575 ; DOI : https:// doi.org/10.4000/cem. 14575

Ce document a été généré automatiquement le 22 septembre 2022.

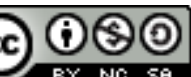

Creative Commons - Attribution - Pas d'Utilisation Commerciale - Partage dans les Mêmes Conditions 4.0 International - CC BY-NC-SA 4.0

https://creativecommons.org/licenses/by-nc-sa/4.0/ 


\section{Vaincre l'enfer sur scène. Les diableries dans le Mystère des Actes des apôtres}

Mémoire de master en histoire et littérature, sous la direction d'Isabel Iribarren et de Thierry Revol. Université de Strasbourg. Soutenu à l'université de Strasbourg le 3 septembre 2015

\section{Mathilde Cornu}

1 Ce mémoire de master s'intéresse aux personnages des diables dans les mystères, en se concentrant sur le rôle qu'ils jouent à la fois dans le salut individuel des différents protagonistes et dans celui des spectateurs qui assistent à ces représentations. Le point de vue adopté propose une analyse davantage doctrinale et historique que littéraire afin de mettre en avant la dimension pastorale de ces scènes visant à l'édification d'un public hétérogène et au contrôle de ses croyances. Le Mystère des Actes des apôtres de Simon Gréban a servi de base à cette étude ${ }^{1}$. Divisée en neuf journées, il s'agit d'une œuvre monumentale composée de plus de 60000 vers, soit le plus colossal à notre connaissance. Ce mystère est ici analysé dans sa version remaniée par Jean Chaponneau, docteur en théologie, en

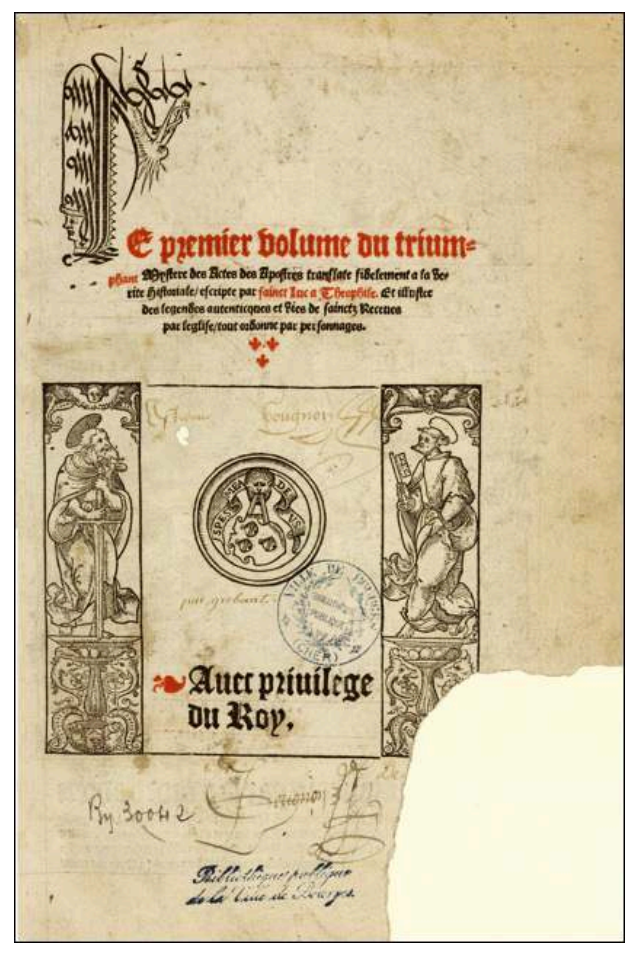
1538 , et qui correspond à sa mise en scène à Bourges en 1536. 
2 L'enfer et les diables apparaissent d'abord visuellement aux spectateurs; la scène comporte diverses mansions qui correspondent aux différents lieux de l'action, encadrées par le Paradis (est) et l'Enfer (ouest). Cette dernière mansion est richement ornée; elle se compose d'une gueule d'enfer et d'une tour centrale entourée de quatre autres tours maçonnées afin de permettre des effets pyrotechniques ${ }^{2}$. Impressionnant par ses décors, l'enfer est par ailleurs peuplé de personnages hauts en couleurs: portant des masques crachant des flammes, l'aspect extérieur des diables indique de prime abord l'importance de ces personnages sur scène, à laquelle corrobore la place croissante qui leur est dédiée dans les textes des mystères, et particulièrement du Mystère des Actes des apôtres ${ }^{3}$.

3 Pourtant, alors qu'ils sont très présents au cours des premières journées, leur rôle s'efface peu à peu au cours des journées suivantes, et leurs scènes n'ont plus de réel lien avec la dramaturgie générale du mystère. Les diables, en effet, perdent peu à peu du terrain dans la lutte qui les oppose à Dieu et aux anges, alors même qu'il s'agit là de leur principale raison d'être. Au cœur de l'enseignement religieux et de la dramaturgie, la lutte qui oppose les puissances célestes et infernales précède les hommes, mais son enjeu est désormais sur terre ${ }^{4}$. Le combat auquel se livrent les forces du bien et celles $\mathrm{du}$ mal concerne directement les spectateurs, et ce dans une perspective eschatologique, car les diables ne peuvent atteindre les anges, et encore moins Dieu, si ce n'est par le sort qui sera réservé aux hommes après leur mort. Dans les mystères de type hagiographiques contrairement à ceux de la Passion, la Rédemption s'est déjà produite, ce qui modifie considérablement l'enjeu dramatique. Cependant, malgré le sacrifice du Christ, l'humanité n'est pas à l'abri du péché et encore moins de l'erreur, et ce sont ces ressorts que vont utiliser les mauvais anges.

Dès lors, la fonction édifiante des diableries va pousser leurs auteurs à mettre en garde le public contre la tentation incitée par ces personnages, dont le seul but est de les mener à la damnation. L'enfer est un lieu où les damnés sont tourmentés sans cesse et effacés de la mémoire divine, où « jamais redemptïon n'entre ». À l'instar des récits de visions, les sévices physiques infligés aux damnés ${ }^{5}$ sont sans cesse décrits et ponctuent les dialogues, notamment à l'arrivée de personnages chez les infernaux. Toutefois, malgré une certaine forme de partage des âmes entre Dieu et les diables, ces derniers échouent toujours dans leurs desseins contre les apôtres: soit leurs projets sont contrecarrés par des personnages positifs, soit ils participent sans en avoir conscience à la réussite de leurs ennemis, principalement lorsqu'ils provoquent le martyr des saints. Les apôtres sauvent de plus en plus d'individus en propageant la foi chrétienne, y compris parmi les alliés des mauvais anges, au grand désespoir de la mesnie infernale. Lors de la seconde journée, un des principaux alliés des diables, le persécuteur de chrétiens Saulus, se convertit et tous les vices de ce personnage font place aux vertus $\mathrm{du}$ futur saint. La conversion est la pire des menaces pour les infernaux, car elle permet même aux personnages les plus négatifs de gagner leur salut; leur espoir de remplir leur habitat avec les juifs et les païens, encore en large majorité, se tarit progressivement.

5 Les diables considèrent le salut des âmes comme un véritable « pillage » de leurs biens de la part de la sphère céleste, cette spoliation de leur droit atteint son sommet à la fin de la quatrième journée, lors de laquelle Michel et Gabriel forcent les portes de l'enfer pour récupérer l'âme du fils de Théophilius. Suite à cet évènement, les diables décident d'endosser le rôle d'avocats et d'intenter un procès à Jésus - qui le premier avait vidé 
leur habitacle - présidé par Justice Divine. Ces personnages prennent volontiers des traits humains que ce soit pour pratiquer la magie cléricale ou pour une reconversion professionnelle sur terre, faute de tâche à accomplir en enfer. En outre, lorsqu'il est question de pallier une conversion ou de s'interroger sur le sort d'une âme, ils sont représentés comme des étudiants de l'Université d'enfer, empruntant le syllabus artien au cours de disputes parodiques qui devaient être tout à fait au goût du public estudiantin ${ }^{6}$. Toutes ces manœuvres qui ont un effet comique indéniable, ne servent encore qu'à insister sur l'échec des infernaux, mauvais avocats et piètres étudiants.

Désespérés, les diables finissent par tenter de ne pas partir en mission, «ce voyage dangereux ». Sathan ${ }^{7}$, qui sait, dès la première journée, que leurs projets à venir échoueront, propose à Astaroth de faire semblant d'être malades pour ne pas être envoyés sur terre. Dès les premières scènes, en effet, il conjecture leur échec final et épouse ainsi le point de vue des spectateurs qui n'ont aucun doute quant à l'issue du mystère. Tous les diables n'ont pas la prescience de Sathan, ce n'est qu'aux dernières diableries qu'ils font en sorte de demeurer en enfer, en prétendant être occupés à torturer des figures mythologiques. Le portrait que l'auteur dresse de la mesnie infernale est donc loin de susciter les craintes. Au contraire, le fait que les êtres qui convoitent leurs âmes soient si faibles et même si proches des hommes a plutôt un effet rassurant.

7 Élyse Dupras ${ }^{8}$ a montré que, comme dans la littérature théologique, les diables, en tant que créatures de Dieu, n'agissent que dans la mesure où ce dernier leur permet; même lorsqu'ils infligent des souffrances aux personnages positifs, ils demeurent soumis au dessein divin. Ces représentations visent à lutter contre le manichéisme et toute tendance chez le peuple d'accorder au diable une puissance considérable, même comparable à celle de Dieu' ${ }^{9}$. Les anges déchus paraissent d'autant moins menaçants que les hommes comptent pour leur protection les anges et la Vierge Marie. Ils affrontent les archanges Michel et Gabriel, dans le contexte spirituel du Moyen Âge tardif, qui voit l'essor de la figure de l'ange gardien : tous les morts qui figurent dans le mystère font l'objet, directement après leur trépas, d'un jugement individuel qui les voue à l'enfer ou au paradis. En ce moment décisif, les diables se tiennent à l'affût, prêt à emmener un nouveau butin, qui, pour les élus, se trouve protégé, souvent par l'archange ${ }^{10}$. Le sentiment de sécurité provoqué par ces personnages déclenche le rire d'un public chrétien face à sa victoire sur l'enfer ${ }^{11}$.

8 Le Mystère des Actes des apôtres raconte la disparition progressive de l'emprise diabolique sur terre grâce à la prédication de ses personnages éponymes. Aussi, dans leurs dernières scènes, les diables ne parlent plus de déjouer les conversions; les âmes de leurs alliés sur terre leur suffisent, quant aux autres, elles sont vouées au paradis, preuve de l'efficacité de la mission apostolique. Le mystère médiéval ne présente pas des diables menaçants, mais quasiment adjuvants. Ils emmènent dans les profondeurs les âmes de ceux qui ne méritent pas le paradis en leur faisant subir des châtiments bien mérités.

9 Les diables, causes des troubles sur terre, participent à la glorification de Dieu par les défaites qu'ils essuient, ils y participent également de façon plus surprenante par des paroles proches des sermons qui enseignent au public comment gagner son salut. Les scènes de diableries contribuent effectivement à rendre les croyances et les pratiques religieuses conformes aux exigences du christianisme tel que l'Église l'entendait, et ce en montrant l'importance non seulement de la miséricorde divine, mais aussi la 
nécessité de la médiation cléricale. Dans la scène du procès de paradis, les diables réclament leurs droits sur les âmes qui leur ont échappé après le sacrifice de Jésus Christ. Cela donne l'occasion à l'auteur d'exposer le dogme chrétien et d'en rejeter les éléments hétérodoxes. Ce passage sert également à expliquer le mystère de la Rédemption. La question qui se pose, en effet, est de savoir si les hommes méritent d'être sauvés, ce que nient les diables, représentés par Sathan. Mais le débat porte également sur la différence entre la rébellion et le péché commis par l'homme et par les mauvais anges envers leur Créateur. Moyse oppose un enseignement angélologique portant sur la nature intellective des anges, qui ont péché en pleine connaissance contre la loi, ce qui les place du côté du péché mortel, provoquant alors une rupture de la grâce divine.

10 Certaines scènes dans lesquelles les anges déchus apparaissent ont même pour vocation d'enseigner aux spectateurs que l'homme en état de péché véniel peut renouer avec Dieu. Au cours de la quatrième journée, afin de préparer convenablement leur procès, Bélial et Sathan consultent un "livre des péchés ", sorte de registre de compte contenant une liste de vices et de comportements proscrits menant à la damnation. En l'ouvrant, ils découvrent avec horreur que «tout est effacé! ». L'abolition des péchés est liée à la contrition et aux larmes versées ${ }^{12}$, comme l'explique Sathan. Elle est d'autant plus primordiale dans un mystère traitant de conversion et proposant aux spectateurs des modèles auxquels s'identifier. Ce même passage, qui rappelle la portée comique des diableries, souligne le rôle du clergé et son importance en tant que médiateur entre l'homme et Dieu. Il apparaît, en effet, que les diables n'ont pas réellement de prise sur les âmes, car Dieu accorde sa miséricorde à ceux qui choisissent de se tourner vers lui. Finalement, pour qu'une âme soit damnée, il faut qu'elle se soit tenue hors de toute possibilité d'être sauvée, donc hors du contrôle clérical, les sacrements conférés par le clergé ayant une valeur salvatrice que les diables ne peuvent que déplorer. Sathan récite même la vulgate des manuels des confesseurs en citant les trois étapes qui mènent à l'horreur du péché, soit à une confession réussie. Ce personnage explique non seulement comment obtenir la rémission des péchés, mais, en plus, il avoue la défaite de son clan grâce à ce même dispositif, que les spectateurs sont bien entendu invités à suivre.

11 Toutefois, certains personnages sont tout de même voués à l'enfer. À l'époque à laquelle l'histoire du Mystère se déroule, le monde est dominé par le paganisme ou par le judaïsme. Les juifs sont les premiers à servir d'appui aux diables; ils apparaissent régulièrement dans la littérature médiévale comme des adversaires désignés des chrétiens. Qualifiés d'« obstinez » à diverses reprises, dans ce mystère, ils préfèrent partir en enfer, plutôt que d'embrasser le christianisme. À côté des juifs, les principaux auxiliaires des diables sont les tyrans et les magiciens. Ces deux figures sont multipliées au cours de l'œuvre, en particulier dans la version du réviseur ${ }^{13}$. Si les premiers sont bien souvent à l'origine du martyr des apôtres, la magie entre en scène à travers leurs suppôts : les magiciens. Ils sont les personnages les plus dangereux non seulement parce que ce sont eux qui vont le plus loin dans l'alliance avec les forces du mal, mais aussi parce que contrairement aux juifs et aux païens, ils ne sont éloignés ni géographiquement ni chronologiquement. Les fatistes, auteurs des mystères, se sont efforcés de montrer au public qu'il devait se méfier à la fois de ces individus et d'une alliance avec les diables, qui n'apporte qu'une satisfaction temporaire, tout en coûtant la damnation éternelle. Enfin, leurs scènes insistent sur la victoire des forces du bien sur la magie inspirée par les diables ${ }^{14}$. Ces alliés subissent en définitive de justes 
châtiments. Leur punition est prononcée par la justice de Dieu à l'encontre de personnages qui se sont volontairement obstinés dans l'erreur et le mal.

Enfin, si l'on se fie à la classification des différentes formes d'infidélités selon Thomas d'Aquin $^{15}$, une autre catégorie de personnages devrait être vouée à l'enfer dans le Mystère, à savoir les païens ${ }^{16}$. Or, il convient de questionner l'application de cette doctrine dans notre Mystère. En effet, à l'instar des visions folkloriques de l'au-delà, mais aussi de la doctrine de l'apocatastase d'origine origéenne, le Mystère des Actes des apôtres semble se rapprocher d'une conception assez large du salut. Le réviseur du Mystère des Actes des apôtres se refuse de condamner à l'enfer le seul païen n'appartenant ni à la catégorie des tyrans, ni à celle des magiciens, mort dans le récit. Il laisse les diablotins Phiton et Panthagruel s'interroger dans un premier temps sur le sort de cette âme - celle du fils de Théophilius - qu'ils ont perdue : Dieu peut-il sauver l'âme d'un païen? A-t-il bénéficié d'une grâce spéciale ? Finalement, son âme n'est pas vouée aux tourments infernaux pour le simple fait qu'il soit païen, car n'ayant pas eu la possibilité de se tourner vers le vrai Dieu. Après avoir été récupérée par les archanges Michel et Gabriel à la prière de l'apôtre Pierre, elle ne va pas au paradis, mais revient sur terre où l'enfant a désormais l'occasion de se tourner vers le christianisme et d'assurer son salut.

Déjà partiellement vaincu dès la mort du Christ, l'enfer est achevé par la prédication des apôtres auxquels les diables ne peuvent faire face. Ridiculisés sur scène, leur pouvoir d'action sur les hommes est limité soit aux infidèles obstinés dans l'erreur, soit, pire, à leurs auxiliaires sur terre. Tous, en plus de leur erreur, commettent de graves péchés, qu'ils réitèrent parfois. Les diables contribuent, par ailleurs, à transmettre aux spectateurs l'image d'un Dieu miséricordieux et à lui enseigner comment renouer avec Dieu et effacer ses péchés sur terre. Ces constats sont à replacer dans le contexte d'un spectacle populaire, car ils amènent à se demander non seulement comment un public chrétien aurait pu se sentir concerné par la damnation, mais aussi si l'enfer du Mystère n'est pas finalement un simple locus demonum ${ }^{17}$.

Reçu : 27 août 2016 - Accepté : 9 décembre 2016

\section{NOTES}

1. SIMON GREBAN, Le Mystère des Actes des apôtres, CNRS-Lamop (UMR 8589) [en ligne: http:// eserve.org.uk/anr].

2. J. тнIBOUST, L'ordre de la triomphante et magnifique monstre du Mystère des saints Actes des apôtres faite à Bourges le dimanche dernier du jour d'avril mil cinq cens trente six, éd. M. LABROUVIE, Bourges, 1836.

3. Raymond Lebègue estime que dans la représentation de 1536, « quinze diables se partageaient

(...) 5489 vers, soit environ neuf pour cent du total », dans R. LEBEGUE, « Le diable dans l'ancien théâtre religieux ", Cahiers de l'Association internationale des études françaises, 3 (1957), p. 97-105, ici p. 100.

4. «Les hommes sont l'enjeu d'un immense combat qui se joue sur terre et ne s'achèvera qu'à la fin du monde ", J. DELUMEAU, Rassurer et protéger : le sentiment de sécurité dans l'Occident d'autrefois, 
Paris, 1989, p. 316. De fait, depuis Grégoire le Grand, l'Église enseigne que Dieu a créé les hommes pour remplir à nouveau les sièges que les diables ont délaissés au paradis au moment de leur révolte.

5. J. FRAPPIER, « Châtiments infernaux et peur du diable d'après quelques textes français du XIII ${ }^{\mathrm{e}}$ et du XIV ${ }^{\mathrm{e}}$ siècle ", Cahiers de l'Association internationale des études françaises, 1/3 (1953), p. 87-96.

6. M. CORNu, « Des diables érudits. Les références intellectuelles dans les diableries du Mystère des Actes des apôtres », Revue des sciences religieuses, 90/3 (2016), p. 315-332.

7. Les noms des personnages du Mystère sont orthographiés de la même manière que dans son édition électronique. Il en va ainsi pour Sathan, Théophilius, Panthagruel et Moyse.

8. E. DUPRAS, Diables des saints. Le rôle des diables dans les mystères hagiographiques français, Genève, 2006.

9. J. LE GOFF, La civilisation de l'Occident médiéval, Paris, 2008.

10. P. FAURE, «Les Cieux ouverts : les anges et leurs images dans le christianisme médiéval. Étude d'anthropologie et d'iconographie religieuse ", Les cahiers du Centre de recherches historiques, 13 (1994), p. 2-10.

11. C. BLuM, «Le diable comme masque. L'évolution de la représentation du diable à la fin du Moyen Âge et au début de la Renaissance ", in M.-T. JONES-DAVIES (dir.), Diable, diables et diableries au temps de la Renaissance, Paris, 1988, p. 149-164.

12. P. NAGY, Le don des larmes au Moyen Âge, Paris, 2000.

13. L'imprimé de 1538 accorde en effet une place plus importante à ces personnages, en ajoutant la scène d'arrivée de Gayus en enfer et en amplifiant le rôle de Néron, allant jusqu'à clore le mystère par son suicide et son arrivée en enfer.

14. Dans notre Mystère, les apôtres affrontent plusieurs magiciens: Hermogenes, Philetus, Arphazat, Zaroes, Baxin et, surtout, Symon Magus, qui est présenté sous les traits de l'Antéchrist. 15. THOMAS D'AQUIN, Somme théologique, II, q. 10, art. 5.

16. R. IMBACH, « Où est cette justice qui les condamne ? Notule sur le sort des païens chez Dante et Thomas d'Aquin », Revue des sciences religieuses, 89/1 (2015), p. 3-23.

17. J. BASCHET, Les justices de l'au-delà. Les représentations de l'enfer en France et en Italie (XIIXIV siècle), Rome, 1993, p. 537-538.

INDEX

Mots-clés : Moyen Âge, théâtre, diables, diableries, enseignement religieux, doctrine, Gréban Simon, Chaponneau Jean, mystère hagiographique, Actes des apôtres

\section{AUTEUR}

\section{MATHILDE CORNU}

Doctorante en linguistique et en histoire au Centre de dialectologie et d'étude du français régional de Neuchâtel et à l'université de Strasbourg 